\title{
Análise do Programa Horizon 2020 da Comunidade Europeia: modelo de eficiência no financiamento à inovação e à geração de riqueza?
}

\author{
Analysis of the European Community Horizon 2020 Program: a model \\ for efficiency in innovation financing and in wealth generation?
}

\author{
Illyushin Zaak Saraiva ${ }^{1}$ \\ Camilo Freddy Mendoza Morejon ${ }^{2}$ \\ Cláudia Crisóstimo ${ }^{3}$ \\ Paulo Rogério de Pinto Rodrigues ${ }^{3}$ \\ ${ }^{1}$ Instituto Federal Catarinense, Luzerna, SC, Brasil \\ ${ }^{2}$ Universidade Estadual do Oeste do Paraná, Toledo, PR, Brasil \\ ${ }^{3}$ Universidade Estadual do Centro-Oeste, Guarapuava, PR, Brasil
}

\begin{abstract}
Resumo
Dada a ausência de estudos brasileiros acerca do programa Horizon 2020 da Comunidade Europeia, com investimento total de 81 bilhões de Euros em CT\&I entre 2014 e 2020, o objetivo do presente trabalho é analisar esse programa, desde a sua origem até o presente momento, destacando as características principais, os resultados, o alcance global, as parcerias brasileiras, além de suas vantagens e oportunidades de melhoria e de desafios. A pesquisa é documental, baseada em artigos e em dados coletados nas bases da Comunidade Europeia que foram tratados no Microsoft Excel, construindo-se gráficos e tabelas sobre os quais realiza-se a análise descritiva. Constata-se que o Horizon 2020, embora tenha encontrado dificuldades e realizado apenas $45 \%$ do orçamento previsto, transcorridos 2/3 da sua vigência, foi capaz de atrair enorme número de propostas de milhares de universidades e instituições, contemplando centenas de países. Desse modo, salienta-se que o programa serviria como modelo no fomento à CT\&I para o Brasil, sugerindo-se para a pesquisa estudos aprofundados sobre sua metodologia.
\end{abstract}

Palavras-chave: Políticas Públicas de CT\&I. Fomento à Inovação. Programa Horizon 2020.

\begin{abstract}
Given the absence of Brazilian studies on the European Community program Horizon 2020, with a total investment of 81 billion Euros in ST\&I between 2014 and 2020, the objective of this work is to analyze the program, from its origin to the present moment, highlighting main characteristics, results, global reach, Brazilian partnerships, as well as its advantages, improvement opportunities, and challenges. The research is documentary, on articles and data collected in the bases of the European Community, being treated in Microsoft Excel, constructing graphs and tables on which descriptive analysis is carried out. Horizon 2020, although encountering difficulties, and achieving only $45 \%$ of its budget, has been able to attract huge numbers of proposals from thousands of universities and institutions, covering hundreds of countries, and it is argued that the program serves as a model in the promotion of ST\&I for Brazil, suggesting to the research in-depth studies on its methodology.
\end{abstract}

Keywords: Public Policies of TC \& I. Fostering of Innovation. Horizon 2020 Program.

Área Tecnológica: Inovação e Desenvolvimento. Economia do Conhecimento. 


\section{Introdução}

Já, desde seus primórdios como ciência estruturada, a Economia buscava compreender a natureza e as causas da riqueza das nações, inicialmente a partir da constatação de que determinados países obtinham graus de produtividade mais elevados que outros, notadamente na manufatura e, em menor grau, na agricultura (SMITH, 1776 [1979]), esforço este que com o desenvolvimento das Ciências Sociais Aplicadas ao longo do século XX, a partir de novas teorias econômicas, se transformou numa busca por compreender em bases socioeconômicas as razões das diferenças no desenvolvimento dos países no mundo capitalista contemporâneo (BRESSER-PEREIRA, 1967). Não se pode nos dias atuais deixar de constatar a correlação prevista pela Teoria do Desenvolvimento entre certas variáveis econômicas como o Estoque de Capital ou, ainda, a Escolaridade Média dos países e a Qualidade de Vida das suas populações, esta última expressa em indicadores como o Índice de Desenvolvimento Humano (IDH) ou o Índice de Felicidade Interna Bruta (FIB) (OLIVEIRA, 2017).

Por outro lado, dadas as características muito peculiares da produção no mundo capitalista a partir dos anos de 1980, com o advento da chamada revolução tecnológica das telecomunicações e da informática e do estabelecimento da chamada economia do conhecimento, concorrem cada vez mais na geração de riqueza e bem-estar em certas regiões algumas características $e$ variáveis relacionadas intrinsecamente com a Pesquisa Científica e com as novas Tecnologias e a Inovação (BRITO CRUZ, 2007) - entendendo-se aqui por inovação não apenas no seu sentido lato de qualquer processo, serviço ou produto cujo estágio atual tenha sido alterado por meio do melhoramento parcial ou total capaz de diferenciá-lo (BACHMANN, 2015), mas também e, principalmente, no sentido mais específico, o de inovação tecnológica - variáveis estas ligadas, por exemplo, à geração de conhecimento: (I) o número de artigos científicos publicados ou, ainda, mais importante e (II) o número de registro de patentes (FERREIRA; GUIMARÃES; CONTADOR, 2009).

Nesse sentido, a geração de conhecimento, no caso o brasileiro, vivenciou nos últimos 20 anos iniciativas governamentais de grande importância, demonstrando mudanças reais na gestão de CT\&I com intuito de estimular o desenvolvimento científico (TURCHI; MORAIS, 2017), como a instituição dos fundos setoriais no final dos anos de 1990, a criação da Lei de Inovação em 2004, a promulgação de incentivos fiscais, por meio da chamada Lei do Bem em 2005, ou, ainda, a instituição do Plano Inova Empresa, em 2013, todos gestados levando-se em consideração iniciativas similares dos chamados países desenvolvidos (DE NEGRI, 2017), mas cujos resultados, considera-se aqui, que ainda precisariam ser apropriadamente avaliados.

Dados preliminares mostram um cenário relativamente positivo no que diz respeito aos resultados dessas políticas pós-2002, quando se observa, por exemplo, o crescimento do número de publicações científicas na primeira década do milênio, período em que o Brasil evoluiu de $1,50 \%$ do total mundial de publicações em 2000 para cerca de 3,00\% em 2010. No que diz respeito, contudo, ao número de depósitos mundiais de patentes, a participação brasileira continua desprezível (BRITO CRUZ, 2007). E, no que diz respeito ao percentual do PIB investido em pesquisa e desenvolvimento ( $\mathrm{P} \& \mathrm{D})$, o crescimento apresentado foi muito modesto, sendo que o investimento privado em P\&D em 2000 foi de 0,49\% do PIB, enquanto em 2011 passou para $0,54 \%$ do PIB, um aumento de apenas $0,06 \%$ em relação ao PIB total (DE NEGRI, 2017). Infelizmente, a partir da crise política de 2015, com o subsequente câmbio governamental via 
impeachment em 2016 e, mais recentemente, com a posse do novo governo eleito, em $1^{\circ}$ de janeiro de 2019, os investimentos federais em Ciência, Tecnologia e Inovação, têm passado por uma onda de ataques e de cortes das verbas para funcionamento das instituições (AGOSTINI, 2019; SALDAÑA, 2020; ZAAK SARAIVA, 2019).

A aparente contradição entre o elevado número de publicações científicas e o baixo número de pedidos de patentes verificados no Brasil, alvo da atenção de bom número de autores e analisada em diversos estudos (BRITO CRUZ, 2007; FERREIRA; GUIMARÃES; CONTADOR, 2009; CRUZ; SOUZA, 2014), quando observada numa perspectiva histórica comparada, mostra uma similaridade entre o cenário brasileiro atual e - guardadas as devidas proporções entre os sistemas de inovação dos dois países e suas economias - o cenário norte-americano pré-1980, momento em que as universidades americanas lideravam a pesquisa básica, mas os resultados científicos dos investimentos em CT\&I não se desdobravam em inovação. Até aquele ano, a legislação dos EUA instituía que o Governo Federal mantivesse a titularidade das descobertas e de inventos relativos aos projetos que financiava, $e$ o impacto industrial da pesquisa foi mínimo (CRUZ; SOUZA, 2014).

Como se sabe, ainda sobre o caso dos Estados Unidos, foi com a adoção de uma nova legislação específica, o Bayh-Dole Act (BDA), que o Estado norte-americano outorgou às universidades de todo o país a titularidade de patentes baseadas nas pesquisas financiadas com recursos federais, possibilitando a elas o licenciamento de suas patentes e o recebimento de royalties, estabelecendo também critérios para repartição dos royalties entre a universidade $e$ os inventores (ALLEN, 2010).

Segundo Cruz e Souza (2014), vários estudos mostram que os resultados do Bayh-Dole Act para a inovação norte-americana - e os resultados de legislações semelhantes adotadas em outros países - modificaram inteiramente o cenário de produção e de difusão tecnológica, permitindo desde 1980 a criação de 4 mil novas companhias a partir de Patentes de Invenção de universidades americanas, sendo que a maioria dessas empresas se localiza próximo aos campi das universidade, tendo a pesquisa acadêmica auxiliado na criação de 1,6 empresas por dia e gerado como resultado econômico bilhões de dólares por meio de contratos de Transferência de Tecnologia (CRUZ; SOUZA, 2014).

O Bayh-Dole Act serviu de inspiração e modelo não apenas para a atual Lei de Inovação brasileira, aprovada em 2004, mas também como inspiração para legislações em diversos países industrializados, além de ser usada como referência para a legislação Europeia de inovação (CRUZ; SOUZA, 2014).

No caso da Europa, e ainda em perspectiva comparada, tem-se por outro lado uma iniciativa de grande importância em termos de investimentos específicos para o desenvolvimento tecnológico, pois o Programa Horizon 2020, mantido pela Comunidade Europeia e voltado ao fomento de Ciência, Tecnologia e Inovação no continente Europeu, se dá por meio do investimento direto de 81 bilhões de Euros em projetos de pesquisa envolvendo empresas e universidades locais e também parceiros em outros continentes (KEUSTERMAN et al., 2018).

Além da importância do Programa Horizon 2020 como modelo de financiamento público em CT\&I, com o qual o Brasil pode buscar aportes e aprendizado, esse programa atualmente também financia diretamente alguns projetos em parceria com universidades brasileiras (EC, 2018), podendo, por isso, ser considerado como uma fonte a mais de recursos para CT\&I 
nacionais, além dos investimentos públicos já realizados anualmente pelas diversas agências governamentais brasileiras.

Apesar das vantagens demonstradas pelo Horizon 2020 no desenvolvimento da Tecnologia e da Inovação no continente Europeu e de atualmente prover montantes substanciais para alguns projetos brasileiros, o programa ainda é relativamente desconhecido no Brasil, inexistindo até o momento bons estudos nacionais acerca dos seus impactos e dos pontos positivos e negativos.

Dessa forma, constitui-se objetivo deste modesto trabalho analisar o Programa Horizon 2020 desde a sua origem até o presente momento, destacando-se suas características principais, seus resultados quantitativos, as parcerias brasileiras do programa e algumas de suas vantagens, oportunidades de melhoria e desafios. O trabalho é constituído de cinco seções: esta introdução, a metodologia, os resultados, as considerações finais e as referências.

\section{Metodologia}

O período aqui analisado é aquele que vai do surgimento dos Framework Programs da Comunidade Europeia, em 1984, até a versão atual, o Programa Horizon 2020, que aqui é analisado desde o seu início em 2014 até dezembro de 2018. Para tanto, foram utilizados dados secundários obtidos por meio de pesquisa documental, focada nos indicadores de desempenho e nos resultados do programa que estão disponíveis nos diversos portais de internet da Comissão Europeia e de outros organismos da Comunidade Europeia, além de alguma literatura acerca da Gestão da Inovação e de Políticas Públicas de Inovação e Transferência de Tecnologia.

Os dados acerca do desempenho do Programa Horizon 2020 são analisados de forma eminentemente quantitativa, por meio de estatística descritiva, utilizando-se o software Microsoft Excel 2013. Para Bogdan e Biklen (1994), assim como para Quivy e Campenhoudt (2008), o método quantitativo basicamente usa três formas de coleta de informações: a) a pesquisa em forma oral ou escrita (entrevistas e questionários); b) a observação; e c) a análise de documentos.

As tabelas disponibilizadas nas diversas bases de dados e websites consultados, quando em formato PDF, foram inicialmente convertidas para o formato Microsoft Word 2013, sendo posteriormente convertidas para o formato Excel, a partir dessa ação foram tratados os dados de acordo com as análises mais adequadas aos objetivos, compondo-se tabelas e gráficos, conforme o tipo de variável e o universo de análise.

\section{Resultados e Discussão}

Com duração de sete anos, de 2014 a 2020, o Horizon 2020 é o maior programa de pesquisa científica e inovação da história da União Europeia (UE), baseando-se nos resultados de programas anteriores, em conjunto com melhorias absorvidas, sendo que o programa financia um total aproximado de 81 bilhões de Euros diretamente do Orçamento da União Europeia, cuja receita é constituída de contribuições nacionais proporcionais ao PIB dos Estados-Membros, além de investimentos privados e de governos nacionais a serem atraídos por meio de parcerias, cujo objetivo final, espera-se, seja transferir dos laboratórios de pesquisa para o mercado diversas descobertas e lançamentos mundiais importantes, capazes de tornar a economia europeia ainda mais competitiva (CE, 2014). 
Inicialmente, é preciso observar-se que o Programa Horizon 2020 é na realidade a continuidade de um programa institucional criado há décadas no seio da Comunidade Europeia, chamado The European Union's Framework Program for Research and Technological Development (EU's FP), no português "Programa-Quadro de Pesquisa e Desenvolvimento Tecnológico da União Europeia" (GALSWORTHY et al., 2012).

O Gráfico 1 apresenta a evolução dos investimentos públicos da Comunidade Europeia, disponibilizados para cada versão do Framework Program (FP) até a versão atual, chamada Horizon 2020.

Gráfico 1 - Investimento da Comunidade Europeia em Ciência, Tecnologia e Inovação nas últimas décadas

\section{Evolução Investimento Total em CT\&I realizado pela Comunidade Europeia por Programa, entre 1984 e 2020 (em € bilhões) \\ Dados coletados em dezembro 2018 \\ * Os dados para o período 2014/2020 são previstos Fontes: CE (2014); GALSWORTHY et AL (2012); PUTSELEVA (2015).}

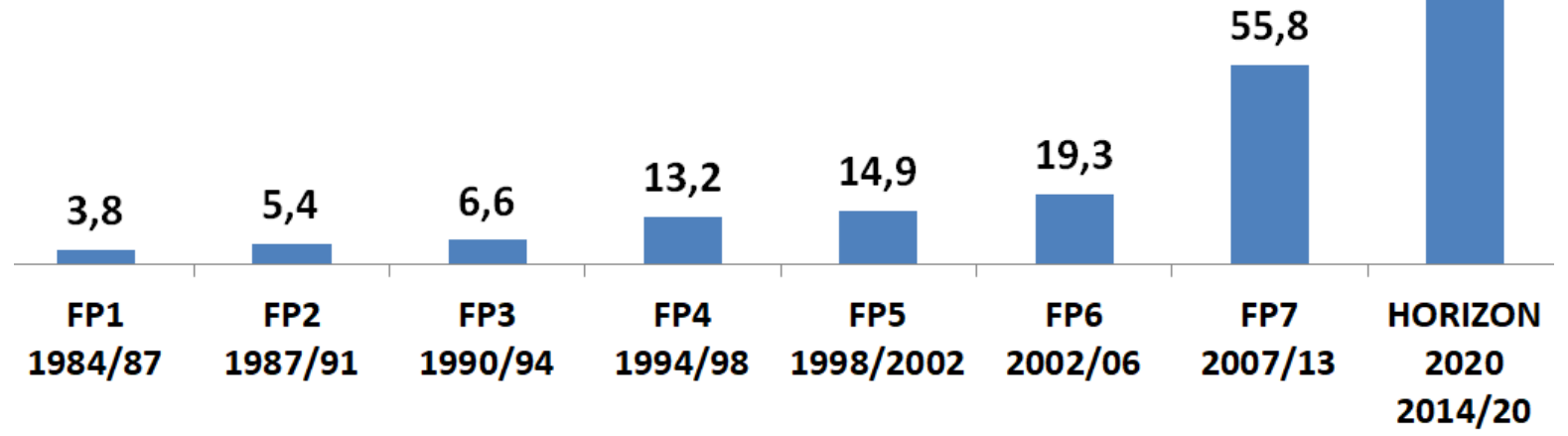

Obs. O montante relativo ao período 2014/2020 é previsto. Até dez. 2018, o programa realizou cerca de €35,56 bilhões.

Fonte: Elaborado pelos autores deste artigo $^{1}$ com dados de CE (2014), Galsworthy et al. (2012), Putseleva (2015)

A mudança representada com o aumento vertiginoso mostrado no Gráfico 1 e, também, com a adoção do nome Horizon 2020, já que até 2006 se adotava simplesmente a nomenclatura FP, não se trata de apenas um simples aumento no interesse dos países da Comunidade Europeia em CT\&I: o programa em seu formato atual, Horizon 2020, apresenta profundas mudanças no que diz respeito aos trâmites burocráticos representados pelo processo de submissão de propostas (CE, 2014).

De acordo com Galsworthy e Mackee (2013), até o FP7, o mecanismo de fomento europeu em CT\&I era extremamente moroso, sendo que as propostas a serem submetidas tinham normalmente várias centenas de páginas, tornando-se, segundo uma anedota comum nas universidades europeias, semelhantes em tamanho às teses de doutorado dos cientistas aplicantes.

Uma das razões dessa mudança foi a de que os chefes de estado europeus, além dos membros do Parlamento Europeu, assumiram a necessidade de remodelar o programa de forma profunda, já que entendiam, pelos resultados apresentados, que o investimento em pesquisa e inovação é crucial para a competitividade da Europa (GALSWORTHY; MACKEE, 2013), e, assim sendo, fixaram o mesmo no centro da estratégia Europa 2020 para um crescimento

\footnotetext{
${ }^{1}$ Os valores aqui apresentados estão dispostos em Euros (€), moeda que só entrou em vigor para transações bancárias (transferências, cheques, etc.) a partir de janeiro de 1999; e na forma de notas e moedas a partir de janeiro de 2002. Todos os valores referentes aos FPs de períodos anteriores foram disponibilizados pela CE já convertidos em Euros.
} 
inteligente, sustentável e inclusivo (CE, 2014). Desde o início do Horizon 2020 até dezembro de 2018 foram contemplados 20.167 projetos, dos quais participam 94.387 integrantes, com uma contribuição efetiva da Comunidade Europeia de 35,56 bilhões de Euros até aquela data.

Entre as instituições científicas/universidades contempladas, a Tabela 1 a seguir apresenta as 20 que receberam os maiores montantes até o momento.

Tabela 1 - As 20 Instituições com maior contribuição financeira do Horizon 2020 até 2018

\begin{tabular}{|c|c|c|}
\hline INSTITUIÇÃO/UNIVERSIDADE & País & Montante $(€)$ \\
\hline Centre National de la Recherche Scientifique CNRS & França & 652.284 .922 \\
\hline Max-Planck-Gesellschaft zur Forderung der Wissenschaften ev & Alemanha & 577.164 .673 \\
\hline Fraunhofer Gesellschaft zur Foerd. der Angewandten Forschung & Alemanha & 367.902 .805 \\
\hline Commissariat al Energie Atomique et aux Energies Alternatives & França & 354.451 .013 \\
\hline The Chancellor Masters and Scholars of the Univ. of Oxford & R. Unido & 307.695 .656 \\
\hline The Chancellor Masters and Scholars of the Univ. of Cambridge & R. Unido & 294.244 .448 \\
\hline University College London & R. Unido & 263.489.302 \\
\hline Imperial College of Science, Technology and Medicine & R. Unido & 198.432 .309 \\
\hline Ecole Polytechnique Federale de Lausanne & Suíça & 191.465.887 \\
\hline Kobenhavns Universitet & Dinamarca & 190.227.690 \\
\hline Katholieke Universiteit Leuven & Bélgica & 186.810.361 \\
\hline Technische Universiteit Delft & Holanda & 183.882.397 \\
\hline The University of Edinburgh & R. Unido & 181.487 .839 \\
\hline Eidgenoessische Technische Hochschule Zuerich & Suíça & 181.035 .984 \\
\hline Agencia Estatal Cons. Superior de Investigaciones Científicas & Espanha & 180.475 .871 \\
\hline Cost Association & Bélgica & 178.169 .171 \\
\hline Consiglio Nazionale delle Ricerche & Itália & 175.342 .495 \\
\hline Deutsches Zentrum fuer Luft - und Raumfahrt ev & Alemanha & 172.166 .990 \\
\hline Institut National de la Sante et de la Recherche Medicale & França & 167.351 .841 \\
\hline The University of Manchester & R. Unido & 137.287.071 \\
\hline TOTAL $=>$ & & 5,14 bi $€$ \\
\hline
\end{tabular}

Fonte: Elaborada pelos autores deste artigo com dados coletados de EC (2018) em 19/12/2018

Com efeito, observa-se que as 20 organizações mais bem contempladas respondem por 5 bilhões de Euros já investidos até dezembro de 2018, ou 14,5\% do total. Chama a atenção a diversidade de países contemplados.

Nesse sentido, o Gráfico 2 apresenta os investimentos efetivamente realizados, desdobrados por cada país executante. 
Gráfico 2 - Investimento realizado pelo Horizon 2020 em Ciência, Tecnologia e Inovação por país

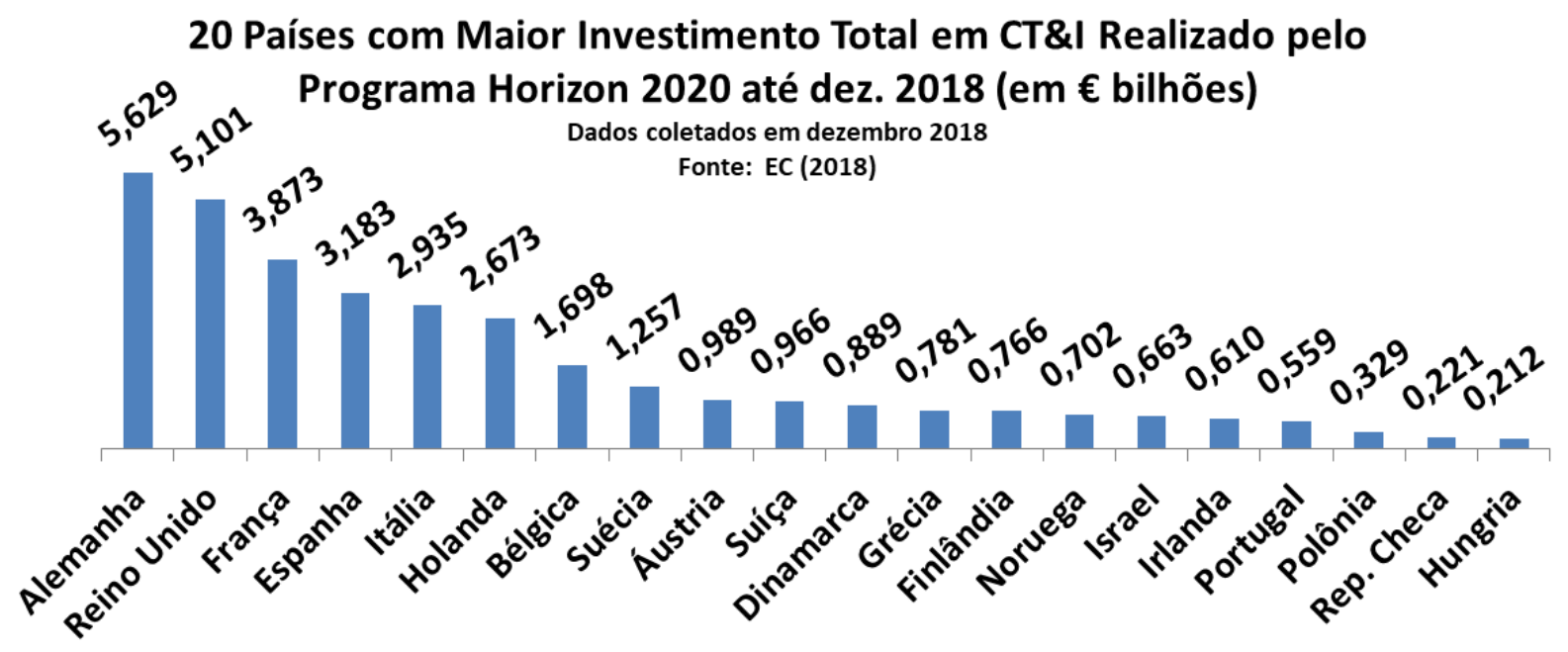

Fonte: Elaborado pelos autores deste artigo com dados de EC (2018)

Diante do fato de que projetos de pesquisa e de inovação financiados pela Comunidade Europeia nas versões anteriores conseguiram unir cientistas e industriais na Europa e no mundo, o Horizon 2020 tem como premissa a participação aberta a empresas, órgãos públicos e instituições de ciência e tecnologia de todo o globo (CE, 2014).

Assim, apresenta-se no Gráfico 3 os investimentos efetivamente realizados até dezembro de 2018, desdobrados por tipo de organização contemplada.

Gráfico 3 - Tipo de organização contemplada versus montante investido pelo Horizon 2020

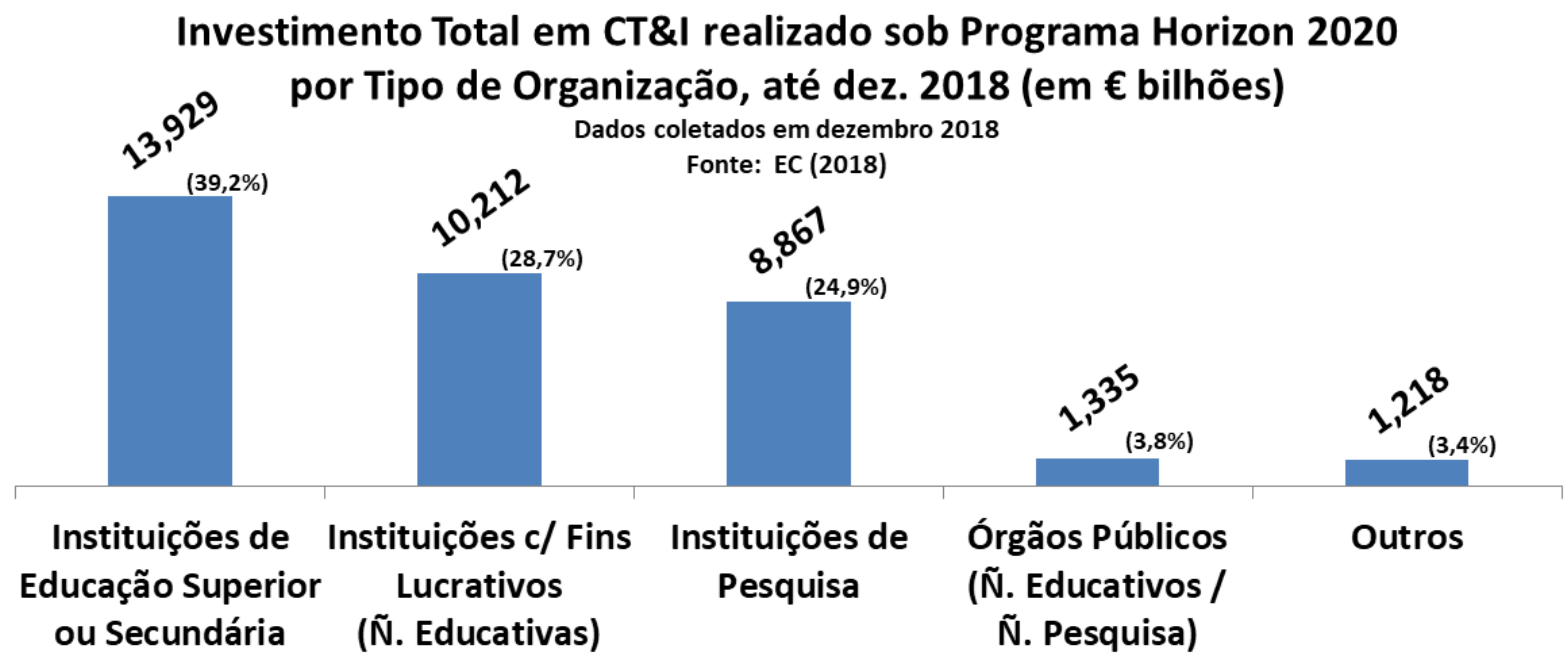

Fonte: Elaborado pelos autores deste artigo com dados de EC (2018)

Observa-se pelos dados apresentados no Gráfico 3 que as organizações lucrativas (empresas) são responsáveis por nada menos que $28,7 \%$ do total de investimentos realizados pelo Horizon 2020 até dezembro de 2018, o equivalente a 10,2 bilhões de Euros.

Contudo, talvez a marca mais importante do programa para o contexto brasileiro, comparando-se com as versões anteriores, seja o número de parceiros no Brasil. A Tabela 2 apresenta as Instituições/Universidades brasileiras contempladas com recursos do Horizon 2020 até dezembro de 2018. 
Tabela 2 - Instituições Brasileiras contempladas com recursos oriundos do Horizon 2020 até 2018

INSTITUIÇÃO/UNIVERSIDADE

Fundação Oswaldo Cruz

Fundação Universidade de Pernambuco

Irmandade da Santa Casa de Misericórdia de São Paulo

Instituto Nacional de Pesquisas Espaciais

Fundação Faculdade de Medicina

Instituto Butantan

Secretaria de Saúde do Estado Ceará

Confederação Nacional da Indústria

Fund. Coord. de Projetos, Pesquisas e estudos Tecnológicos Coppetec

Universidade de São Paulo

Conselho nacional das Fundações de Estaduais de Amparo à Pesquisa

Universidade Estadual de Campinas

Universidade Federal da Bahia

Universidade Federal de Minas Gerais

Fundação de Amparo a Pesquisa do estado de São Paulo

Financiadora de Estudos e Projetos

Ass. Técnico Científica Estudo Colaborativo L.

Americano de Malformação Congênita

Núcleo de Gestão do Porto Digital

Ass. Nacional de Pesquisa e Desenvolvimento das Empresas Inovadoras - Anpei

União Brasileira de Educação e Assistência Associação

Ass. Brasileira das Instituições de Pesquisa Tecnológica e Inovação

Fundação de Apoio à Universidade de São Paulo

Fundação de Desenvolvimento da Unicamp-Funcamp

Fundação Bahiana de Infectologia

Universidade Federal do Pará

Agencia Paulista de Tecnologia dos Agronegócios

Universidade Federal do Rio de Janeiro

Universidade Federal de Goiás

Laboratório Nacional de Computação Científica
Montante $(€)$

$1.351 .993 €$

$1.244 .671 €$

$813.255 €$

$812.171 €$

$471.437 €$

$454.688 €$

$394.480 €$

$392.750 €$

$344.164 €$

$306.250 €$

$302.438 €$

$281.625 €$

$255.882 €$

$225.000 €$

$198.313 €$

$198.295 €$

$191.150 €$

$137.841 €$

$137.625 €$

$136.625 €$

$131.396 €$

$100.076 €$

$95.769 €$

$70.481 €$

$60.500 €$

$60.000 €$

$38.498 €$

$8.125 €$

$6.250 €$

TOTAL $=>$

9.221.748 $€$

Fonte: Elaborada pelos autores deste artigo com dados coletados de EC (2018) em 19/12/2018

Embora o montante investido pelo Horizon 2020 em instituições brasileiras até dezembro de 2018, da ordem dos 9 milhões de Euros, possa ser considerado desprezível diante do total 
de 35 bilhões de Euros já realizado em todo o mundo até a data, observa-se na Tabela 2 que há razoável diversidade e boa descentralização geográfica, especialmente quando verificada a região de localização das instituições contempladas.

Com efeito, entre os 9 milhões de Euros recebidos pelas organizações brasileiras, pode-se observar que há instituições das Regiões Nordeste, Sudeste, Centro-Oeste e Norte do Brasil, além de organismos nacionais como o Confap, o INPE e a Fiocruz.

Também chama atenção o elevado montante que algumas organizações obtiveram por meio do Horizon 2020, como no caso da Fundação Oswaldo Cruz, da Fundação Universidade de Pernambuco, da Irmandade da Santa Casa de Misericórdia de São Paulo e do Instituto Nacional de Pesquisas Espaciais, com cerca de 1 milhão de Euros cada um.

Além disso, pode-se inferir que, dados alguns valores extremamente baixos, como aqueles obtidos pela Universidade Federal de Goiás e pelo Laboratório Nacional de Computação Científica, de menos de 10 mil Euros cada um, é possível até mesmo, aos pesquisadores de centros mais afastados, e com projetos relativamente simples, participarem do Horizon 2020 com chances reais de obter financiamento para suas pesquisas.

Ainda acerca da participação de organizações brasileiras no Horizon 2020, destaca-se finalmente que o baixo número de instituições brasileiras provavelmente tem relação com a falta de informação acerca da existência do programa dentro da comunidade científica ou, ainda, o desconhecimento de que esse programa é aberto a instituições do Brasil, sugerindo-se aos órgãos de fomento nacionais e regionais uma divulgação mais completa e a realização de número satisfatório de workshops com pró-reitores ou coordenadores de pesquisa.

Dos resultados apresentados até o momento, pode-se inferir algumas das grandes virtudes do Horizon 2020, como o fato de que o programa contemplou projetos de mais de 100 países, originários de milhares de instituições, com presença marcante de universidades de ponta, mas também com uma participativa expressão de organizações não educacionais privadas, responsáveis por quase $30 \%$ dos valores investidos.

Os dados disponíveis acerca dos investimentos já realizados pelo programa, especialmente quando observados sob o viés da Estratégia Nacional de Ciência, Tecnologia e Inovação (ENCTI) do Brasil, mostram, assim, um quadro relativamente diferente do brasileiro, no qual as universidades públicas parecem ser as organizações mais preparadas, já que predominam como destinação dos investimentos governamentais em CT\&I.

Outra grande diferença do Horizon 2020 em relação à ENCTI, já observada por Squeff, Alves e Barcelos (2017), é que enquanto o programa europeu foi criado por meio de uma normativa do próprio Parlamento Europeu, o que garante a ele o caráter de plano governamental e o selo de instrumento para a implementação financeira, com orçamento definido e instrumentos previamente estabelecidos, a estratégia brasileira, por outro lado, foi criada como apenas um documento balizador elaborado pelo então Ministério de Ciência, Tecnologia, Inovações e Comunicações.

Finalmente, no que diz respeito às oportunidades de melhoria do Programa Horizon 2020, destaca-se as observações de Keusterman et al. (2018) no sentido de garantir que os projetos aprovados atinjam o impacto previsto, tendo em vista a observação de órgãos e de universidades no sentido de que o impacto alcançado pelos investimentos nos diversos projetos finalmente aprovados, foi muitas vezes menor do que o impacto previsto no momento da avaliação desses mesmo projetos de inovação. 


\section{Considerações Finais}

O objetivo deste trabalho foi analisar o programa de fomento à CT\&I chamado Horizon 2020, desde sua origem em 2014 até o presente momento, destacando-se suas características principais, resultados, parcerias brasileiras do programa e algumas de suas vantagens, oportunidades de melhoria e desafios.

Foram coletadas informações em bases disponíveis nos diversos portais da Comunidade Europeia, a partir dos quais se compôs um banco de dados simplificado, a partir do qual os dados foram tratados quantitativamente no software Microsoft Excel, sendo então construídos gráficos e tabelas sobre os quais se realizou a análise apresentada.

Observou-se que o Horizon 2020 se trata de um programa continental de proporções gigantescas, cujo orçamento para o período de vigência, 2014 a 2020, é de pouco mais de 81 bilhões de Euros. Além disso, a legislação e as normas europeias para concessão dos investimentos foram inteiramente revistas para facilitar a submissão de propostas. A legislação Europeia para transferência de tecnologia - assim como a legislação brasileira implantada a partir da Lei da Inovação de 2004 - sofreu influência do Bayh Dole Act, com foco no estímulo para a transferência de tecnologia pela concessão de patentes às universidades e aos pesquisadores contemplados com investimentos públicos.

Mas o que se destaca no caso do programa europeu é a grande responsabilidade assumida pelos Estados componentes da União Europeia no fomento à inovação.

Constata-se que o Programa Horizon 2020, embora tenha apresentado até dezembro de 2018 algumas dificuldades, como o fato de ter realizado apenas $45 \%$ do orçamento total, tendo já transcorrido 2/3 do seu período de vigência, mostrou-se capaz de atrair um grande número de propostas de milhares de universidades e instituições, contemplando centenas de países. Por isso, acredita-se e defende-se aqui que o Horizon 2020 deva servir como modelo para futuros programas brasileiros de fomento à CT\&I.

No que diz respeito aos pontos de melhoria do programa, é possível destacar as observações de Keusterman et al. (2018), no sentido de se incluir ferramentas e processos para garantir que, em sua próxima versão, o impacto previsto nos projetos de inovação aprovados seja realmente alcançado no final.

Dessa forma, considera-se que o objetivo deste trabalho foi cumprido, sugerindo-se para pesquisas posteriores a realização de estudos mais aprofundados sobre os resultados do programa Horizon 2020 em termos qualitativos e, principalmente, sobre as características de sua metodologia - e as adaptações necessárias - que poderiam ser aplicadas no Brasil.

\section{Agradecimentos}

Registra-se aqui o mais profundo agradecimento ao Instituto Federal Catarinense pela bolsa de afastamento para pós-graduação do primeiro autor, sem a qual este e outros trabalhos não poderiam ter sido concluídos. 


\section{Referências}

AGOSTINI, Renata. MEC Cortará Verba de Universidade por 'Balbúrdia' e já enquadra UnB, UFF e UFBA. Estadão, São Paulo, 30 abr. 2019. Disponível em: https://educacao.estadao.com.br/noticias/ geral,mec-cortara-verba-de-universidade-porbalburdia-e-ja-mira-unb-uff-e-ufba,70002809579. Acesso em: 3 maio 2019.

ALLEN, Joseph. The Enactment of Bayh-Dole, An Inside Perspective. IP Watchdog, [S.1.], 28 nov. 2010. Disponível em: http://www.ipwatchdog.com/2010/11/28/the-enactment-of-bayh-dole-an-insideperspective/id=13442/. Acesso em: 20 dez. 2018.

BACHMANN, Dórian L. Guia para Inovação: instrumento para a melhoria das dimensões da inovação. 2. ed. Curitiba: SK Editora Ltda., 2015.

BOGDAN, R. C.; BIKLEN, S. K. Investigação qualitativa em educação: uma introdução à teoria e aos métodos. Porto: Porto Editora, 1994.

BRESSER-PEREIRA, Luiz Carlos. A teoria econômica e os países subdesenvolvidos. Revista de Administração de Empresas, São Paulo, v. 7, n. 24, julho-setembro, 1967. Disponível em: www. scielo.br/scielo.php?script=sci_arttext\&pid=S0034-75901967000300001. Acesso em: 16 nov. 2018.

BRITO CRUZ, Carlos H. Ciência e Tecnologia no Brasil. Revista USP, São Paulo, n. 73, p. 58-90, março-maio, 2007. Disponível em: https://www.revistas.usp.br/revusp/article/download/13589/15407/0. Acesso em: 16 nov. 2018.

CE - COMISSÃO EUROPEIA. Horizon 2020 em breves palavras: o programa quadro de investigação e inovação da UE. Luxemburgo: Serviço das Publicações das Comunidades Europeias, 2014. 40 p. ISBN 978-92-79-38928-3. Disponível em: https://ec.europa.eu/programmes/horizon2020/ sites/horizon2020/files/H2020_PT_KI0213413PTN.pdf. Acesso em: 16 nov. 2018.

CRUZ, Hélio Nogueira; SOUZA, Ricardo Fasti. Sistema Nacional de Inovação e a Lei da Inovação: Análise Comparativa entre o Bayh-Dole Act e a Lei da Inovação Tecnológica. Revista de Administração e Inovação, São Paulo, v. 11, n. 4, p. 329-354, out.-dez. 2014. Disponível em: https://ac.els-cdn.com/S180920391630208X/1-s2.0-S180920391630208X-main.pdf?_tid=f5404515-e4b34325-9080-5eeafc91453d\&acdnat=1545334746_6ef89d69da278b053cc62d13f5f41c24. Acesso em: 16 nov. 2018.

DE NEGRI, Fernanda. Por uma nova geração de políticas de inovação no Brasil. In: TURCHI, Lenita Maria; MORAIS, J. Mauro. (org.). Políticas de apoio à inovação Tecnológica no Brasil: avanços recentes, limitações e propostas de ações. Brasília: IPEA, 2017. p. 25-46. Disponível em: http://repositorio.ipea.gov.br/bitstream/11058/8125/1/Políticas\%20de\%20apoio\%20à\%20inovação\%20 tecnológica\%20no\%20Brasil.pdf. Acesso em: 16 nov. 2017.

EC - EUROPEAN COMMISSION. H2020 Projects - Summary. Atualizado até outubro de 2018. Disponível em: https://webgate.ec.europa.eu/dashboard/sense/app/e8a41234-20b4-4e7e-80ef335dd9e6ae36/sheet/941d3afe-da24-4c2e-99eb-b7fcbd8529ee/state/analysis. Acesso em: 19 dez. 2018.

FERREIRA, Ademir Antônio; GUIMARÃES, Edílson Rodrigues; CONTADOR, José Celso. Patente como instrumento competitivo e como fonte de informação tecnológica. Revista Gestão e

Produção, São Carlos, v. 16, no. 2, p. 209-221, abr.-jun. 2009. Disponível em: www.scielo.br/pdf/gp/ v16n2/v16n2a05.pdf. Acesso em: 16 nov. 2018. 
GALSWORTHY, Michael J. et al. Academic output of 9 years of EU investment into health research. The Lancet, [S.I.], v. 380, edição 9.846, p. 971-972, setembro de 2012. Disponível em: https://www. thelancet.com/journals/lancet/article/PIIS0140-6736(12)61528-1/fulltext. Acesso em: 16 nov. 2018.

GALSWORTHY, Michael; MACKEE, Martin. Europe's 'Horizon 2020' science funding programme: how is it shaping up? Journal of Health Services Research \& Policy, [S.l.], v. 18, n. 3, p. 182185, Jul. 2013. DOI: 10.1177/1355819613476017. Disponível em: https://www.ncbi.nlm.nih.gov/ pmc/articles/PMC4107840/. Acesso em: 16 nov. 2018.

KEUSTERMAN, Laura et al. Impact and the next Framework Programme for Research and Innovation (FP9): note from the League of European Research Universities. Abr. 2018. Disponível em: https://www.leru.org/files/Publications/Impact-and-the-next-Framework-Programme-for-Researchand-Innovation.pdf. Acesso em: 16 nov. 2018.

OLIVEIRA, Pedro Cavalcante. O que diferencia países pobres das nações ricas? Mercado Popular - Economia, [S.l.], 21 de janeiro de 2017. Disponível em: http://mercadopopular.org/2017/01/o-quediferencia-paises-pobres-das-nacoes-ricas/. Acesso em: 16 nov. 2018.

PUTSELEVA, Maria. From "FP" to Horizon 2020: opportunities within EU Framework Programmes for RTD. 2015. Disponível em: https://slideplayer.com/slide/3304146/. Acesso em: 16 nov. 2018.

QUIVY, R.; CAMPENHOUDT, L. V. Manual de investigação em ciências sociais. 5. ed. Lisboa: Gradiva, 2008.

SALDAÑA. Paulo. MEC Estende Corte de 30\% de Verbas a Todas as Universidades Federais. Folha de São Paulo. 30 abr. 2019. Disponível em: https://www1.folha.uol.com.br/educacao/2019/04/mecestende-corte-de-30-de-verbas-a-todas-universidades-federais.shtml. Acesso em: 3 maio 2019.

SMITH, Adam. Investigação sobre a natureza e as causas da riqueza das nações. São Paulo: Abril Cultural, (1776 [1979]). ISBN: 85-351-0827-0.

SQUEFF, Flávia de Holanda Schmidt; ALVES, Camila; BARCELOS, Catarina. Como a União Europeia Financia a Pesquisa? Radar IPEA, [S.1.], n. 50, IPEA, 2017. Disponível em: http:// repositorio.ipea.gov.br/bitstream/11058/7837/1/Radar_n50_como_a_União.pdf. Acesso em: 16 nov. 2018.

TURCHI, Lenita Maria; MORAIS, José Mauro. Introdução. In: TURCHI, Lenita Maria; MORAIS, José Mauro (org.). Políticas de apoio à inovação Tecnológica no Brasil: avanços recentes, limitações e propostas de ações. Brasília, DF: IPEA, 2017. p. 9-21. Disponível em: http://repositorio.ipea.gov.br/ bitstream/11058/8125/1/Políticas\%20de\%20apoio\%20à\%20inovação\%20tecnológica\%20no\%20Brasil. pdf. Acesso em: 16 nov. 2018.

ZAAK SARAIVA, Illyushin. Elementos para Análise do Ataque Falacioso contra Universidades e Serviço Público no Neoliberalismo Brasileiro pós-2015: Balbúrdia, homens-pauta-bomba, ataque e recuo. Observatorio de la Economía Latinoamericana, junio 2019. Disponível em: https://doi. org/10.13140/RG.2.2.25333.63209/2. Acesso em: 6 jul. 2019. 


\section{Sobre os Autores}

\section{Illyushin Zaak Saraiva}

E-mail: illyushin.saraiva@ifc.edu.br Especialista em Educação Empreendedora pela UFSJ em 2014.

Endereço profissional: Instituto Federal Catarinense, Campus Luzerna, Rua Vigário Frei João, n. 550, Luzerna, SC. CEP: 89609-000.

\section{Camilo Freddy Mendoza Morejon}

E-mail: camilo_freddy@hotmail.com

Doutor em Engenharia Mecânica pela UFRJ em 2003.

Endereço profissional: Universidade Estadual do Oeste do Paraná, Centro de Engenharia e Ciências Exatas, Rua da Faculdade, n. 645, Jardim La Salle, Toledo, PR. CEP: 85903-000.

\section{Cláudia Crisóstimo}

E-mail: crisostimoadv@gmail.com

Mestra em Direito pela UFPR em 2008.

Endereço profissional: Universidade Estadual do Centro-Oeste, Agência de Inovação Tecnológica de Guarapuava. Campus Cedeteg, Rua Simeão Varela de Sá, n. 3, Vila Carli, Guarapuava, PR. CEP: 85040-080.

\section{Paulo Rogério de Pinto Rodrigues}

E-mail: prprodrigues@gmail.com

Doutor em Química pela USP em 1997.

Endereço profissional: Universidade Estadual do Centro-Oeste, Agência de Inovação Tecnológica de Guarapuava. Campus Cedeteg, Rua Simeão Varela de Sá, n. 3, Vila Carli, Guarapuava, PR. CEP: 85040-080. 